\title{
The feasibility and safety of percutaneous dilatational tracheostomy without endotracheal guidance in the intensive care unit
}

\author{
Ji Eun Kim', Dong Hyun Lee ${ }^{2}$ \\ Departments of ${ }^{1}$ Emergency Medicine and ${ }^{2}$ Intensive Care Medicine, Dong-A University Hospital, Dong-A University College of Medicine, Busan, Korea
}

Background: Percutaneous dilatational tracheostomy (PDT) is a common procedure in intensive care units (ICUs). Although it is thought to be safe and easily performed at the bedside, PDT usually requires endotracheal guidance, such as bronchoscopy. Here, we assessed the clinical outcomes and safety of PDT conducted without endotracheal guidance.

Methods: In the ICU and coronary ICU at a tertiary hospital, PDT was routinely performed without endotracheal guidance by a single medical intensivist using the Griggs technique PDT kit (Portex Percutaneous Tracheostomy Kit). We retrospectively reviewed the electronic medical records of patients who underwent PDT without endotracheal guidance.

Results: From January 1 to December 31, 2018, 78 patients underwent PDT without endotracheal guidance in the ICU and coronary ICU. The mean age of these subjects was $71.9 \pm 11.5$ years, and 29 (37.2\%) were female. The mean Acute Physiology and Chronic Health Evaluation (APACHE) II score was 25.9 \pm 5.8 . Fifty patients (64.1\%) were on mechanical ventilation during PDT. Failure of the initial PDT attempt occurred in four patients (5.1\%). In two of them, PDT was aborted and converted to surgical tracheostomy; in the other two patients, PDT was reattempted after endotracheal reintubation, with success. Minor bleeding at the tracheostomy site requiring gauze changes was observed in five patients (6.4\%). There were no airway problems requiring therapeutic interventions or procedure-related sequelae.

Conclusions: PDT without endotracheal guidance can be considered safe and feasible.

Key Words: airway management; intensive care units; tracheostomy

\section{INTRODUCTION}

Percutaneous dilatational tracheostomy (PDT) is commonly performed for the airway management of critically ill patients in the intensive care unit (ICU) [1-3]. Although the timing of tracheostomy in ventilated patients is a controversial topic [4,5], tracheostomy has many advantages in facilitating weaning, improving patient mobilization and reducing sedative requirements, laryngeal damage, breathing effort, and costs [6,7].

Although surgical tracheostomy is the routine practice of choice, PDT is also widely performed in the ICU $[1,3]$. Endoscopic guidance with bronchoscopy was the standard of prac-

\section{Original Article}

Received: June 28, 2021

Revised: September 10, 2021

Accepted: September 14, 2021

Corresponding author
Dong Hyun Lee
Department of Intensive Care
Medicine, Dong-A University
Hospital, 26 Daesingongwon-ro,
Seo-gu, Busan 49201, Korea
Tel: +82-51-240-2922
E-mail: icu1@dau.ac.kr

E-mail: icu1@dau.ac.kr

Copyright (C) 2022 The Korean Society of Critical Care Medicine

This is an Open Access article distributed under the terms of Creative Attributions Non-Commercial License (https:// creativecommons.org/li-censes/by-nc/4.0/ which permits unrestricted noncommercial use, distribution, and reproduction in any medium, provided the original work is properly cited. 
tice at initial introduction because of its direct visualization of the endotracheal position [8]. Physicians have performed PDT by a variety of endotracheal guides, such as bronchoscopy [9], real-time ultrasound $[10,11]$, and light sources [12]. In a recent study, PDT was safely performed by experienced physicians both with and without bronchoscopic guidance [13]. Additionally, Pattnaik et al. [14] reported a case series of 300 patients who safely underwent PDT without bronchoscopic guidance. The purpose of this study was to analyze the feasibility and safety of PDT without endotracheal guidance at the bedside in the ICU.

\section{MATERIALS AND METHODS}

\section{Research Ethics}

This study was approved by the Institutional Review Board of Dong-A University Hospital (IRB No. DAUHIRB-20-036). The Board waived the requirement for informed consent due to the retrospective study design.

\section{Study Design and Patient Selection}

This was a retrospective observational study of patients who underwent PDT without endotracheal guidance at a single tertiary hospital from January 1 to December 31, 2018. Patients who underwent PDT were initially screened, and the corresponding electronic medical records, including procedure notes, nurse notes, vital signs, and physician progression notes, were manually reviewed.

Patients $\geq 18$ years of age were included if they underwent PDT without endotracheal guidance, which was an almost routine practice for tracheostomy during the period. Patients were excluded if PDT was performed by other physicians or by endotracheal guidance (bronchoscopy, video laryngoscopy, ultrasound, etc.) and if they initially underwent surgical tracheostomy.

\section{Outcome Measurements}

The primary outcome was defined as successful PDT. PDT failure was defined as reintubation or airway management using a supraglottic airway device (SAD) for any reason or conversion to surgical tracheostomy after the initiation of PDT. Secondary outcomes were defined as immediate (within 24 hours) and delayed (within 7 days) procedure-related complications, such as hypoxia, bleeding and hypotension within 24 hours after PDT and delayed problems associated with tracheostomy within 7 days after PDT. Hypotension was defined as a systolic

\section{KEY MESSAGES}

- Percutaneous dilatational tracheostomy (PDT), which is accepted to be safe, usually performed with endotracheal guidance.

- PDT without endotracheal guidance can also be performed safely.

blood pressure of $70 \mathrm{~mm} \mathrm{Hg}$ or less; major bleeding was defined as bleeding requiring an aggressive intervention, such as embolization, electrocoagulation, and wound revision; and minor bleeding was defined as hemorrhage requiring direct compression at the procedure site within an hour. Hypoxia was defined an oxygen saturation on pulse oximetry of $88 \%$. Survival and discharge were also reviewed.

\section{Procedure}

PDT was routinely performed by a single medical intensivist using the Griggs technique PDT kit (Portex Percutaneous Tracheostomy Kit; Smith Medical, Kent, England) without any endotracheal guidance. The physician in this study, as a medical intensivist, had experience with more than 100 cases of PDT for more than 3 years and performed PDT with bronchoscopic endotracheal guidance in 20 cases and without endotracheal guidance in 52 cases in the previous 1 year.

The PDT procedures were routinely performed as follows. The patient was prepared by the administration of analgesics and sedatives and was placed in the supine position with the neck hyperextended. Continuous remifentanil infusion from $0.06 \mu \mathrm{g} / \mathrm{kg} / \mathrm{hr}$ to $0.14 \mu \mathrm{g} / \mathrm{kg} / \mathrm{hr}$ was used for analgesia, and bolus $5 \mathrm{mg}$ of midazolam was used for procedural sedation. A neuromuscular blockade agent was not used during PDT. Blood pressure, heart rate and pulse oximetry were continuously monitored. The neck of the patient was widely disinfected with chlorhexidine gluconate solution and covered with sterile drapes for the aseptic procedure. The operator palpated the neck carefully from top to bottom to identify two important landmarks: the cricothyroid membrane and the sternal notch. The incision site was placed under 1 fingerbreadth from the cricothyroid membrane, targeting between the 2nd and 3rd tracheal cartilaginous rings. The sternal notch was a landmark warning not to proceed below this line. Local anesthesia with $2 \%$ lidocaine and 1:100,000 epinephrine was given subcutaneously at the incision site. After making an approximately 2-cm transverse skin incision, dissection of the pretracheal soft tissue was performed. The assistant changed the fraction of in- 
spired oxygen setting to $100 \%$ and withdrew the endotracheal tube to $15 \mathrm{~cm}$ on the incisor while the operator palpated the tracheal membrane at the insertion site. Puncture of the trachea was cautiously performed on the midline of the incision, and the endotracheal position was confirmed by air bubble aspiration of a syringe containing $0.9 \%$ normal saline. Then, a guide wire was inserted, followed by removal of the needle. The initial stoma was made using a dilator, followed by second stoma formation with dilating forceps by the Griggs technique. Then, the tracheostomy cannula was placed using the Seldinger technique along the guide wire, and the patient was totally extubated by the assistant. The proper endotracheal position of the tracheostomy tube confirmed the smooth passage of the rubber suction tip into the cannula. During the procedure, most events concerning the patient were recorded on the electronic medical record system. All patients were confirmed by chest $\mathrm{X}$-rays, and vital signs were recorded after the procedure.

\section{Statistical Analysis}

The data were analyzed using IBM SPSS ver. 21.0 (IBM Corp., Armonk, NY, USA) for Windows. Nominal variables were examined by the Fisher's exact test, and continuous variables were examined by the Shapiro-Wilk and the Kolmogorov-Smirnov test for assessing the normality of the distribution. If the test result was significant $(\mathrm{P}<0.05)$, the sample data distribution was considered non-normal. Over half of the continuous variables followed a normal distribution and are presented as the mean and standard variation. The other continuous variables followed a non-normal distribution and are presented as the median (interquartile range). Nominal variables are presented as the number and incidence rate (\%). Values were rounded off to one decimal place.

\section{RESULTS}

\section{Patient Characteristics}

From January 1 to December 31, 2018, 78 patients underwent PDT in the ICU and coronary ICU. The mean age was $71.9 \pm 11.5$ years, and 29 patients (37.2\%) were female. More than half of the patients were admitted to pulmonology (46 patients, 59\%), followed by cardiology ( 7 patients, $9 \%$ ), nephrology ( 5 patients, $6.4 \%$ ), and others (16 patients, 20.5\%). The mean Acute Physiology and Chronic Health Evaluation (APACHE) II score was $25.9 \pm 5.8$, and 71 patients (91\%) had received mechanical ventilation during their whole hospitalization. Fifty patients (64.1\%) had been on mechanical ventilation during PDT; ventilator modes were synchronized intermittent mandatory ventilation (35 patients, $44.9 \%$ ), pressure support ventilation (9 patients, 11.5\%), and controlled mandatory ventilation (6 patients, 7.7\%). Their mean $\mathrm{FiO}_{2}$ was $40.0 \%$ (30.0-40.0), and the duration from mechanical ventilation to PDT was $14.0 \pm 7.5$ days. Seventeen patients (21.8\%) needed vasopressors to maintain their blood pressure. Thirty patients (38.5\%) had taken anticoagulants or antiplatelets within 7 days before PDT, and their mean duration was 2.0 days (2.0-3.3 days): aspirin (9 patients, 11.5\%), clopidogrel (9 patients, $11.5 \%)$, enoxaparin (14 patients, 17.9\%), heparin (3 patients, 3.8\%), warfarin (3 patients, $3.8 \%$ ), and dalteparin (1 patient, $1.3 \%$ ). Detailed baseline characteristics of the patients are shown in Table 1.

\section{Successful PDT and Complications Associated with PDT Failure}

The primary outcome of the study was successful PDT. Among the 78 study patients, successful PDT was achieved in 74 $(94.9 \%)$ at the first cannulation attempt. Four patients experienced initial cannulation failure. In two of these patients, a

Table 1. Characteristics of patients who underwent PDT without endotracheal guidance

\begin{tabular}{lc}
\hline Characteristics & Value $(\mathrm{n}=78)$ \\
\hline Age (yr) & $71.9 \pm 11.5$ \\
Sex & \\
$\quad$ Male & $49(62.8)$ \\
$\quad$ Female & $29(37.2)$ \\
Height (cm) & $163.3 \pm 9.6$ \\
Weight (kg) & $56.4 \pm 12.1$ \\
Admission department & \\
$\quad$ Pulmonology & $46(59)$ \\
$\quad$ Cardiology & $7(9)$ \\
$\quad$ Nephrology & $5(6.4)$ \\
Gastroenterology & $4(5.1)$ \\
Other & $16(20.5)$ \\
APACHE II score & $25.9 \pm 5.8$ \\
Mechanical ventilation during PDT & \\
On mechanical ventilation & $50(64.1)$ \\
Mean FiO & (\%) (n=50) \\
Duration from mechanical ventilation to PDT (day) & $40(30-40)$ \\
Vasopressor therapy & $14.0 \pm 7.5$ \\
Anticoagulant/antiplatelet treatment within 7 days & $17(21.8)$ \\
Anticoagulation duration before PDT (day) & $30(38.5)$ \\
\hline
\end{tabular}

Values are presented as mean \pm standard deviation, number (\%), or median (interquartile range).

PDT: percutaneous dilatational tracheostomy; APACHE: Acute Physiology and Chronic Health Evaluation. 
SAD was used to maintain airway patency, followed by successful PDT. The other two patients were reintubated and converted to surgical tracheostomy. All of the patients recovered completely after PDT or surgical tracheostomy without any hypoxia-related sequelae.

In the assessment of secondary outcomes, hypotension was the most common complication (16 patients, 20.5\%); eight patients $(10.3 \%)$ required crystalloid replacement only, four patients (5.1\%) required vasopressors, and four patients (5.1\%) required both crystalloid replacement and vasopressors. Minor bleeding at the tracheostomy site occurred in five patients (6.4\%), and they needed only supportive care, such as direct manual compression or simple dressing. Major bleeding requiring surgical intervention or transfusion was not reported (Table 2). There were no early complications concerning the airway requiring therapeutic procedures or interventions within a week after the procedure.

After PDT, 47 patients (60.3\%) were transferred to another hospital, five patients (6.4\%) were discharged home, and 26 patients (33.3\%) died. The mean time from the day of PDT to death was 23.0 days (11.3-67.0 days) (Table 3 ).

\section{Clinical Characteristics of Patients with PDT Failure on the First Attempt}

Four patients failed at the first attempt of PDT due to hypoxia. Clinical characteristics of patients with initial PDT failure are described in Table 4. Four patients experienced failed weaning from mechanical ventilation for various reasons and underwent PDT after different durations of mechanical ventilation. In the first case of failure, a 65-year-old woman with nonsmall-cell lung carcinoma needed mechanical ventilation due to consistent atelectasis and pneumonia. The 1st PDT attempt

Table 2. Early complications of PDT without endotracheal guidance

\begin{tabular}{lc}
\hline Complication & Value $(n=78)$ \\
\hline Hypoxia & $4(5.2)$ \\
Conversion to surgical tracheostomy & $2(2.6)$ \\
Subglottic airway device insertion & $2(2.6)$ \\
Hypotension & $16(20.5)$ \\
Crystalloid replacement only & $8(10.3)$ \\
Vasopressor treatment only & $4(5.1)$ \\
Both crystalloid replacement and vasopressor therapy & $4(5.1)$ \\
Bleeding & $5(6.4)$ \\
Major bleeding & 0 \\
Minor bleeding & $5(6.4)$ \\
\hline
\end{tabular}

Values are presented as number (\%).

PDT: percutaneous dilatational tracheostomy. failed, and the $\mathrm{SpO}_{2}$ dropped to $67 \%$ during the 1st attempt. A SAD was inserted for oxygenation, and the 2nd PDT attempt was successful. In the second case, a 59-year-old man with olivopontocerebellar atrophy required long-term mechanical ventilation due to postoperative complications of gastric ulcer perforation. After the 1st PDT attempt, the mechanical ventilator was connected, but the tidal volume was not received from the mechanical ventilator due to tracheostomy site obstruction. The patient's $\mathrm{SpO}_{2}$ dropped to $76 \%$, and a $\mathrm{SAD}$ was inserted immediately; the 2nd PDT attempt was successful. In the third case, a 48-year-old woman with alcoholic liver cirrhosis failed to wean from mechanical ventilation after liver transplantation. The 1st PDT attempt failed, and the $\mathrm{SpO}_{2}$ dropped to $75 \%$. The patient was reintubated and underwent conversion to surgical tracheostomy. Lastly, an 84-year-old man with hypertension and diabetes mellitus underwent PDT because of poor airway protection after spinal stenosis surgery. The 1st PDT attempt failed, and the $\mathrm{SpO}_{2}$ dropped to $79 \%$. A SAD was inserted, and the subsequent 2 nd attempt failed. This patient also underwent conversion to elective surgical tracheostomy.

\section{DISCUSSION}

In the present retrospective observational study, most patients (94.9\%) underwent successful PDT without endotracheal guidance. Although four patients experienced hypoxia requiring reintubation or SADs during the procedure, there were no hypoxia-related sequelae.

The initially introduced standard PDT procedure included endotracheal guidance using bronchoscopy [15]. However, bronchoscopy requires an experienced and professional physician with additional assistants. Devices and equipment for PDT should also include a bronchoscope. To overcome the complexity and high degree of the required labor force, physicians have developed alternative endotracheal guides, such as ultrasound [10,11] and light sources [12]. Finally, PDT without endotracheal guidance has also been introduced $[13,14]$.

Table 3. Outcome of patients who underwent PDT without endotracheal guidance

\begin{tabular}{lc}
\hline Outcome & Value $(n=78)$ \\
Discharged to home & $5(6.4)$ \\
Transferred to another hospital & $47(60.3)$ \\
Expired & $26(33.3)$ \\
\hline
\end{tabular}

Values are presented as number (\%).

PDT: percutaneous dilatational tracheostomy. 
Table 4. Characteristics of patients with PDT failure on the first attempt

\begin{tabular}{|c|c|c|c|c|c|c|}
\hline $\begin{array}{l}\text { Age }(y r) / \\
\text { sex }\end{array}$ & Underlying disease & Reason for MV & $\begin{array}{l}\text { Duration from } \\
\text { MV to PDT (day) }\end{array}$ & PDT course & $\begin{array}{c}\text { Complications during } \\
\text { PDT }\end{array}$ & Special note \\
\hline $65 / F$ & $\begin{array}{l}\text { NSCLC } \\
\text { (adenocarcinoma) }\end{array}$ & Pneumonia, atelectasis & 15 & $\begin{array}{l}\text { 1st attempt failure } \\
\text { 2nd attempt success }\end{array}$ & Hypoxia $\left(\mathrm{SpO}_{2}, 67 \%\right)$ & \\
\hline $59 / \mathrm{M}$ & $\begin{array}{l}\text { Olivopontocerebellar } \\
\text { atrophy }\end{array}$ & $\begin{array}{l}\text { Postoperative care } \\
\text { (gastric ulcer perforation) }\end{array}$ & 19 & $\begin{array}{l}\text { 1st attempt failure } \\
\text { 2nd attempt success }\end{array}$ & $\begin{array}{l}\text { Hypoxia }\left(\mathrm{SpO}_{2}, 76 \%\right) \\
\text { Tracheostomy site } \\
\text { obstruction }\end{array}$ & Heparinization for CRRT \\
\hline $48 / F$ & $\begin{array}{l}\text { Alcoholic liver } \\
\text { cirrhosis }\end{array}$ & $\begin{array}{l}\text { Postoperative care } \\
\text { (liver transplantation) }\end{array}$ & 23 & $\begin{array}{l}\text { 1st attempt failure } \\
\text { 2nd surgical tracheostomy }\end{array}$ & $\begin{array}{l}\text { Hypoxia }\left(\mathrm{SpO}_{2}, 75 \%\right) \\
\text { Bloody secretion from } \\
\text { E-tube }\end{array}$ & $\begin{array}{l}\text { Thrombocytopenia } \\
\text { (platelet count, } 38,000 / \mu l)\end{array}$ \\
\hline $84 / \mathrm{M}$ & $\begin{array}{l}\text { Hypertension, } \\
\text { diabetes mellitus }\end{array}$ & $\begin{array}{l}\text { Postoperative care } \\
\text { (spinal stenosis) }\end{array}$ & 20 & $\begin{array}{l}\text { 1st attempt failure } \\
\text { 2nd attempt failure } \\
\text { 3rd surgical tracheostomy }\end{array}$ & Hypoxia $\left(\mathrm{SpO}_{2}, 79 \%\right)$ & \\
\hline
\end{tabular}

PDT: percutaneous dilatational tracheostomy; MV: mechanical ventilation; NSCLC: non-small-cell lung carcinoma; CRRT: continuous renal replacement therapy; E-tube: endotracheal tube.

Recently, PDT has been considered safe, but procedures requiring advanced airway management are often dangerous and devastating and may even lead to death. Common immediate or early complications occurring within 7 days are bleeding, hypoxia, posterior tracheal wall injury, tracheal tube obstruction, and so on. In the present study, one of the early complications was hypotension (16 patients, $20.5 \%$ ), which is thought to be associated with sedative use. Hypoxia (4 patients, $5.1 \%$ ) and bleeding (5 patients, 6.4\%) were also reported. Hypotension and bleeding were minor events that resolved by conservative management. Major surgical problems, such as tracheal wall injury and tube obstruction, were not reported.

Although four patients required airway management because of hypoxia during PDT, normal range oxygenation was recovered after proper airway management without any hypoxia-related sequelae. However, conversions to surgical tracheostomy were required in two patients (2.6\%). Beyond 7 days, late complications such as subglottic or tracheal stenosis, unplanned decannulation, stomal infection, and permanent voice changes can occur $[16,17]$. However, in this study, severe late complications in need of medical attention were not reported.

In the present study, remifentanil and midazolam were routinely used for procedural analgesia and sedation. Although hypoxia was observed in $5.1 \%$ of patients, apnea was not observed. Due to endotracheal intubation with or without mechanical ventilation, most patients were receiving an continuous remifentanil infusion titrated to minimize pain and agitation before PDT. As a result, patients received relatively high doses of remifentanil via continuous infusion and an additional bolus of midazolam for the PDT procedure without apnea event. The higher incidence of hypotension (20.5\%) than hypoxia without any apnea event can be explained by the basal opioid exposure of the patients.

Previous studies on PDT without endotracheal guidance found that the procedure was relatively safe. However, Pattnaik et al. [14] reported several important complications in 300 cases of PDT without endotracheal guidance, including two cases $(0.6 \%)$ of false lumen insertion, two cases $(0.6 \%)$ of major bleeding requiring transfusion, and two cases $(0.6 \%)$ of death within 24 hours as early complications. Additionally, four cases (1.3\%) of subglottic stenosis were also reported as late complications. Jackson et al. [13] compared the outcomes of PDT with or without bronchoscopy guidance in trauma patients. There was no significant difference in the incidence of major early and late complications. One case of cardiac arrest was reported in the PDT with bronchoscopy guide group.

In this study, the patients received PDT without endoscopic guidance in the ICU. The use of endotracheal guides facilitates a safe and precise procedure [9-11], but this usually requires considerable technician labor, costs, and time. Furthermore, in many hospitals, bronchoscopy and real-time ultrasound are not always accessible for tracheostomy, and physicians must wait for the devices and technicians to be prepared. On the other hand, clinicians performing PDT without endotracheal guidance do not need to wait for devices or technicians, and the procedure can be performed immediately if the patient and operating physician are ready. The present investigation reports a lower incidence of major complications than previous studies.

The authors also tried to discriminate risk factors to predict PDT failure. Although previous studies have reported PDT 
without endoscopic guidance to be safe, they have also reported fatal complications, such as false-lumen insertion, major bleeding, and cardiac arrest $[13,14]$. However, there are no available data on clinical factors associated with failure or fatal complications. In the presenting study, we also failed to discriminate the risk factors for the failure in the first PDT attempt.

The present investigation has several limitations. First, it was a retrospective observational study with a small number of patients in a single tertiary medical center. However, most patients were homogeneous; most were treated in the medical department, and PDT was performed by a single intensivist. Second, the study did not include patients who initially underwent surgical tracheostomy, suggesting selection bias. There is a possibility that those who underwent initial surgical tracheostomy exhibited a difficult anatomy for tracheostomy. Third, an experienced single medical intensivist performed PDT in the ICU. The physician had good performance in this study, but the proficiency of the operator was the key point in performing PDT without endotracheal guidance. Thus, there is the possibility that safety is not guaranteed in PDT depending on the physician's ability.

In conclusion, PDT without endotracheal guidance can be performed safely on most ICU patients. Regarding the costs, time, and requirement of specific technicians for endotracheal guidance, PDT performed by experienced physicians using precise landmarks can be a feasible choice. Further studies comparing PDT with and without endotracheal guidance can yield precise clinical guidance.

\section{CONFLICT OF INTEREST}

No potential conflict of interest relevant to this article was reported.

\section{ORCID}

Ji Eun Kim https://orcid.org/0000-0002-7067-7899

Dong Hyun Lee https://orcid.org/0000-0001-6253-3396

\section{AUTHOR CONTRIBUTIONS}

Conceptualization: DHL. Data curation: DHL. Formal analysis: JEK. Methodology: all authors. Project administration: all authors. Visualization: all authors. Writing-original draft: JEK. Writing-review and editing: all authors.

\section{REFERENCES}

1. Freeman BD, Isabella K, Lin N, Buchman TG. A meta-analysis of prospective trials comparing percutaneous and surgical tracheostomy in critically ill patients. Chest 2000;118:1412-8.

2. Brambrink A. Percutaneous dilatation tracheostomy: which technique is the best for the critically ill patient, and how can we gather further scientific evidence? Crit Care 2004;8:319-21.

3. Cooper RM. Use and safety of percutaneous tracheostomy in intensive care: report of a postal survey of ICU practice. Anaesthesia 1998;53:1209-12.

4. Hyde GA, Savage SA, Zarzaur BL, Hart-Hyde JE, Schaefer CB, Croce MA, et al. Early tracheostomy in trauma patients saves time and money. Injury 2015;46:110-4.

5. Terragni PP, Antonelli M, Fumagalli R, Faggiano C, Berardino M, Pallavicini FB, et al. Early vs late tracheotomy for prevention of pneumonia in mechanically ventilated adult ICU patients: a randomized controlled trial. JAMA 2010;303:1483-9.

6. Flaatten H, Gjerde S, Heimdal JH, Aardal S. The effect of tracheostomy on outcome in intensive care unit patients. Acta Anaesthesiol Scand 2006;50:92-8.

7. Ciaglia P, Firsching R, Syniec C. Elective percutaneous dilatational tracheostomy: a new simple bedside procedure; preliminary report. Chest 1985;87:715-9.

8. Hinerman R, Alvarez F, Keller CA. Outcome of bedside percutaneous tracheostomy with bronchoscopic guidance. Intensive Care Med 2000;26:1850-6.

9. Gadkaree SK, Schwartz D, Gerold K, Kim Y. Use of bronchoscopy in percutaneous dilational tracheostomy. JAMA Otolaryngol Head Neck Surg 2016;142:143-9.

10. Rudas M, Seppelt I, Herkes R, Hislop R, Rajbhandari D, Weisbrodt L. Traditional landmark versus ultrasound guided tracheal puncture during percutaneous dilatational tracheostomy in adult intensive care patients: a randomised controlled trial. Crit Care 2014;18:514.

11. Gobatto AL, Besen BA, Cestari M, Pelosi P, Malbouisson LM Ultrasound-guided percutaneous dilational tracheostomy: a systematic review of randomized controlled trials and meta-analysis. J Intensive Care Med 2020;35:445-52.

12. Baek JK, Lee JS, Kang M, Choi NJ, Hong SK. Feasibility of percutaneous dilatational tracheostomy with a light source in the surgical intensive care unit. Acute Crit Care 2018;33:89-94.

13. Jackson LS, Davis JW, Kaups KL, Sue LP, Wolfe MM, Bilello JF, et al. Percutaneous tracheostomy: to bronch or not to bronch-that is the question. J Trauma 2011;71:1553-6.

14. Pattnaik SK, Ray B, Sinha S. Griggs percutaneous tracheostomy 
without bronchoscopic guidance is a safe method: a case series of 300 patients in a tertiary care intensive care unit. Indian J Crit Care Med 2014;18:778-82.

15. Ciaglia P. Technique, complications, and improvements in percutaneous dilatational tracheostomy. Chest 1999;115:1229-30.
16. Mehta C, Mehta Y. Percutaneous tracheostomy. Ann Card Anaesth 2017;20(Suppl 1):S19-25.

17. Fernandez-Bussy S, Mahajan B, Folch E, Caviedes I, Guerrero J, Majid A. Tracheostomy tube placement: early and late complications. J Bronchology Interv Pulmonol 2015;22:357-64. 